\title{
SPECTRUM OF BALLOONING INSTABILITIES IN A STELLARATOR
}

W.A. Cooper, D.B. Singleton, R.L. Dewar

Submitted for publication in Physics of Plasmas 


\title{
Spectrum of ballooning instabilities in a stellarator
}

\author{
W. A. Cooper (cooper@crppsun1.epH.ch) \\ Centre de Recherche en Physique des Plasmas, \\ Ecole Polytechnique Fédérale de Lausanne, \\ 21, av. des Bains, \\ CH-1007 Lausanne, Switzerland \\ D. B. Singleton (David.Singleton@anu.edu.au) \\ ANU Supercomputer Facility, \\ The Australian National University, \\ Canberra, A.C.T. 0200, Australia \\ R. L. Dewar (Robert.Dewar@anu.edu.au) \\ Plasma Research Laboratory \& Department of Theoretical Physics, \\ Research School of Physical Sciences and Engineering, \\ The Australian National University, \\ Canberra, A.C.T. 0200, Australia
}

(August 22, 1995)

\begin{abstract}
The recent revival of interest in the application of the "ballooning formalism" to low-frequency plasma instabilities has prompted a comparison of the Wentzel-Brillouin-Kramers (WKB) ballooning approximation with an (in principle) exact normal mode calculation for a three-dimensional plasma equilibrium. Semiclassical quantization, using the ideal magnetohydrodynamic (MHD) ballooning eigenvalue to provide a local dispersion relation, is applied
\end{abstract}


to a ten-field period stellarator test case. Excellent qualitative agreement, and good quantitative agreement is found with predictions from the TERPSICHORE code for toroidal mode numbers from 1 to 14 and radial mode numbers from 0 to 2 . The continuum bands predicted from three-dimensional WKB theory are too narrow to resolve.

52.35.Py, 52.65.+z, 52.55.Hc 


\section{INTRODUCTION}

The ballooning formalism ${ }^{1}$ is often regarded as applying only to tokamak modes with very large toroidal mode number $n$. This is for two reasons, the first being that $n^{-1}$ (or $\left.n^{-1 / 2}\right)$ is used as an asymptotic expansion parameter. The second reason is that, at least in ideal MHD, the highest growth rates for ballooning modes occur in the limit $n \rightarrow \infty$, when the modes become localized about the most unstable magnetic surface.

However, such localized modes cannot have much effect on turbulent transport. This has prompted recent interest ${ }^{2,3}$ in extending the formalism to treat drift waves with more extended radial structure and finite $n$ by use of the WKB-ballooning theory. This has also led to controversy $y^{4,5}$ over the nature of the normal mode spectrum - whether it is discrete or continuous. It has also been suggested ${ }^{6}$ that the validity of the ballooning theory for moderate $n$ is questionable.

There is little formal difference between WKB-ballooning theory as applied to drift waves and to ideal MHD modes, the main difference being that, in the drift-wave case, turning points will in general be complex. Thus, for assessing the practical applicability of WKBballooning for calculating approximate global eigenmodes at moderate $n$, it is sufficient in the first instance to use the simpler MHD model.

The formal ballooning theory ${ }^{7,8}$ is based on standard asymptotic analysis, in which one orders $k_{\|} / k_{\perp}=O(\epsilon)$, where $k_{\|}$and $k_{\perp}$ are the projections of the local wavevector respectively parallel and perpendicular to the magnetic field $B$. However, since it is not practicable to make a formal estimate of the residual error when the expansion is, as always, truncated at the lowest nontrivial order, one must resort to comparison with numerical solutions in order to get a feel for the typical domain of applicability. This is particularly the case for the WKB method, which typically works well beyond the range one might expect on the basis of formal asymptotics.

Such a comparison was in fact done in Ref. 7 for a critical $\beta$ (plasma pressure/magnetic pressure) study of two tokamak equilibrium sequences. It was found that WKB-ballooning 
theory worked well for $n \gtrsim 5$, but one equilibrium sequence showed oscillations for $n \lesssim 5$ which were not explainable on the basis of simple semiclassical quantization for "trapped modes," i.e. modes whose ray paths are restricted to a finite interval (width $<2 \pi$ ) in the ballooning parameter $\theta_{k}$.

These oscillations may be explained ${ }^{9}$ as interference fringes due to coupling, via tunneling, between adjacent trapped modes lying close to the separatrix dividing trapped and "passing" modes, i.e. modes whose ray paths are unbounded in $\theta_{k}$. For slightly lower $n$, degenerate passing modes lying on either side of the "island" can also couple. These passing modes are the ones recently singled out as being more dangerous for anomalous transport ${ }^{2,3}$ due to finite radial extent, although trapped modes close to the separatrix have comparable extent. The treatment of trapped and passing modes, and their coupling, may be effected by a new ballooning formalism" based on a "twisted radial Fourier transform." ${ }^{10}$ In this paper we restrict attention to trapped modes.

In view of the recent interest in finite- $n$ ballooning, we have thought it useful to perform a new ideal MHD numerical comparison study. The present study differs from that in Ref. 7 in that (a) it uses a more realistic model for the kinetic energy and calculates growth rates for a single equilbrium, rather than critical $\beta_{8}$ for a sequence; (b) it finds several of the infinite number of radial eigenmodes predicted from the semiclassical quantization formula for trapped modes; ${ }^{7}$ and (c) it treats a nonaxisymmetric equilibrium so that qualitatively new features of the WKB ray tracing problem in such geometries ${ }^{8}$ might potentially be observed.

The equilibrium chosen for study, a ten-field-period torsatron with a $\beta$ well beyond criticality for ballooning instability, turns out in fact to behave in some ways similarly to an axisymmetric case. The present study is not principally concerned with nonaxisymmetric effects, but the torsatron case will be useful for comparison with more strongly nonaxisymmetric devices such as heliacs.

The paper is arranged as follows. In Sec. II the WKB-ballooning formalism is reviewed, while in Sec. III its implementation on a parallel computer is described. Section IV 
describes the equilibrium, and compares the calculation of global eigenmodes using the TERPSICHORE ${ }^{11}$ code with those found from the WKB-ballooning formalism, followed by Conclusions.

\section{WKB-BALLOONING FORMALISM}

We assume the magnetic field $B(r)$ to be integrable, that is the field lines all lie within nested invariant tori, or magnetic surfaces, which are labeled by the enclosed poloidal flux $2 \pi \psi$. Introducing straight-field-line poloidal and toroidal angles $\theta$ and $\zeta$, and a Clebsch potential

$$
\alpha=\zeta-q \theta
$$

where $q$ is the safety factor (tokamaks) or inverse rotational transform (stellarators), we have

$$
B=\nabla \alpha \times \nabla \psi
$$

Then, following Dewar and Glasser, ${ }^{8}$ we write the fluid displacement as

$$
\xi=\hat{\xi}(r, \epsilon) \exp \left[i \epsilon^{-1} S(r)-i \omega t\right]
$$

where $\hat{\xi}$ is slowly varying and the eikonal $S \equiv S(\alpha, \psi)$, so that the local wavevector

$$
k \equiv \nabla S \equiv k_{\alpha} \nabla \alpha+k_{q} \nabla q \equiv k_{\alpha}\left(\nabla \alpha+\theta_{k} \nabla q\right)
$$

lies in the plane transverse to $B$ at each point. Here the ballooning parameter $\theta_{k}$ appears as the ratio $k_{q} / k_{\alpha}$, revealing its dual role as a dimensionless radial wavenumber ${ }^{7}$ and an angle. The formal expansion parameter $\epsilon$ expresses the assumed smallness of the perpendicular wavelength (i.e. $k / \epsilon$ is large). The parallel wavenumber $k_{\|}$does not appear explicitly in this formalism, slow phase variations along $B$ being absorbed into $\xi$.

To derive the ballooning mode equation, we apply the ballooning formalism to the energy principle. ${ }^{12}$ The internal potential energy can be expressed $a s^{13}$ 


$$
\delta W_{p}=\frac{1}{2} \int d^{3} x\left[|C|^{2}+\Gamma p|\nabla \cdot \xi|^{2}+D|\xi \cdot \nabla q|^{2}\right]
$$

where the modified perturbed magnetic field is

$$
C=\nabla \times(\xi \times B)+\frac{j \times \nabla q}{|\nabla q|^{2}} \xi \cdot \nabla q,
$$

the MHD driving term is

$$
D=2 \frac{j \times \nabla q}{|\nabla q|^{2}} \cdot(B \cdot \nabla)\left(\frac{\nabla q}{|\nabla q|^{2}}\right),
$$

$j$ is the current density field, $\Gamma$ is the adiabatic index and $p$ is the thermal pressure.

In the standard ideal MHD model, the kinetic energy is expressed as

$$
\delta W_{k}=\frac{\omega^{2}}{2} \int d^{3} x \rho_{M} \xi^{2},
$$

and the stability is determined by solving $\delta W_{p}+\delta W_{k}=0$. The Euler-Lagrange equation derived from the minimization of the energy principle after the application of the 3D ballooning mode formalism results in a fourth order ordinary differential equation (ODE) on each magnetic field line. ${ }^{8}$ However, the MHD model can be simplified further by adopting a kinetic energy model that eliminates the sound wave continuous spectrum from the problem. This reduces the ballooning equation to a second order ODE. ${ }^{1}$ One form that was adopted in the CAS3D code ${ }^{14}$ and in an early version of the TERPSICHORE code ${ }^{11}$ is to consider the kinetic energy as the unit matrix. Another more physically realistic alternative is to express the mass density as a dyadic tensor ${ }^{15}$ so that the kinetic energy is written as

$$
\delta W_{k}=\frac{\omega^{2}}{2} \int d^{3} x \xi \cdot \rho_{M} \cdot \xi
$$

In the PEST2 code, ${ }^{15}$ the mass density dyadic is $\rho_{M}=\nabla \psi \nabla \psi$ so that only the radial component of the displacement vector contributes to the kinetic energy. In the TERPSICHORE code, the mass density dyadic is

$$
\begin{aligned}
\rho_{M}= & {\left[2 \Phi^{\prime}(s)\right]^{2} \nabla s \nabla s } \\
& +(\sqrt{g})^{2}[B \times(\nabla \theta \times \nabla \zeta)][B \times(\nabla \theta \times \nabla \zeta)],
\end{aligned}
$$


where $\sqrt{g}$ is the Jacobian of the transformation from Cartesian coordinates to magnetic coordinates, and the toroidal magnetic flux is $2 \pi \Phi$, which we treat as a function of the radial variable $s \equiv \Phi(\psi) / \Phi\left(\psi_{a}\right)$ normalized to unity at the plasma edge $\psi=\psi_{a}$. This choice for $\rho_{M}$ annihilates the parallel component of the displacement vector in the kinetic energy, partly because the radial variable $s$ is a surface quantity. This then allows the plasma compression term $\Gamma_{p}|\nabla \cdot \xi|^{2}$ of the potential energy to be eliminated algebraically from the energy principle. The eigenvalues, however, cease to correspond to the physical growth rates of the instability when an artifical mass tensor is used, but the points of marginality remain unaffected. For the numerical evaluation of global stability, the simplifications invoked to describe the kinetic energy translate into a reduction of the unknown components of the displacement vector to be computed from three to two and in a significant suppression of the spectral broadening of the Fourier components of the perturbation caused by the coupling to the sound waves. ${ }^{16}$

The application of the ballooning formalism to the energy principle with the TERPSICHORE kinetic energy norm reduces the local MHD stability problem to the second order ODE eigenvalue problem, involving the scalar amplitude variable $\widehat{\xi} \equiv \widehat{\xi} \cdot B \times \nabla S /|\nabla S|^{2}$ as dependent variable and $\theta$ as independent variable,

$$
\begin{aligned}
& \frac{\partial}{\partial \theta}[ {\left.\left[C_{p}+C_{s}\left(\theta-\theta_{k}\right)+C_{q}\left(\theta-\theta_{k}\right)^{2}\right] \frac{\partial \hat{\xi}}{\partial \theta}\right] } \\
&+ {\left[d_{p}+d_{s}\left(\theta-\theta_{k}\right)\right] \hat{\xi} } \\
&-\omega^{2} \sqrt{g}\left[[2 q(s)]^{2}+\left[q^{\prime}(s)\left(\theta-\theta_{k}\right)\right]^{2}\right] \hat{\xi}=0,
\end{aligned}
$$

when expressed in Boozer magnetic coordinates. ${ }^{17}$ The coefficients are ${ }^{18}$

$$
\begin{aligned}
C_{p} & =\left(\frac{g_{s s}}{\sqrt{g}}-\frac{B_{s}^{2}}{\sqrt{g} B^{2}}\right), \\
C_{.} & =2 \frac{q^{\prime}(s)}{q(s)}\left[\frac{J(s) B_{s}}{\sqrt{g} B^{2}}-\frac{g_{s \theta}}{\sqrt{g} B^{2}}\right], \\
C_{q} & =\frac{\left[q^{\prime}(s) \psi^{\prime}(s)\right]^{2}}{\sqrt{g} B^{2}}|\nabla s|^{2}, \\
d_{p} & =\frac{p^{\prime}(s)}{\psi^{\prime}(s)}\left[\frac{\sqrt{g} p^{\prime}(s)}{B^{2}}+\frac{J(s) \psi^{\prime \prime}(s)-I(s) \Phi^{\prime \prime}(s)}{B^{2}}\right.
\end{aligned}
$$




$$
\begin{array}{r}
\left.-\frac{\partial \sqrt{g}}{\partial s}+\sqrt{g} B \cdot \nabla\left(\frac{B_{a}}{B^{2}}\right)\right], \\
d_{s}=-\frac{q^{\prime}(s)}{\psi^{\prime}(s)} \sqrt{g} B \cdot \nabla\left(\frac{j \cdot B}{B^{2}}\right),
\end{array}
$$

where $g_{i j}$ are the lower metric elements, $B$, is the radial component of the magnetic field in the covariant representation that is obtained from the MHD equilibrium force balance relation $^{13}$

$$
\sqrt{g} B \cdot \nabla B_{c}=\sqrt{g} p^{\prime}(s)+\psi^{\prime}(s) J^{\prime}(s)-\Phi^{\prime}(s) I^{\prime}(s)
$$

the toroidal current flux is $2 \pi J$, the poloidal current flux is $2 \pi I$, and prime' indicates the derivative of a flux surface quantity with respect to $s$. The choice of magnetic coordinates is motivated by the field lines being straight and by the correspondence of the poloidal and toroidal components of the magnetic field, in the covariant representation, with the current flux functions $J(s)$ and $-I(s)$, respectively, which facilitates the evaluation of the parallel current density.

The ballooning equation, Eq. (11), is an eigenvalue equation under the boundary condition that $\hat{\xi}$ vanishes as $\theta \rightarrow \pm \infty$. Taking $\omega^{2}$ to be the eigenvalue we replace it with the symbol $\lambda$ and solve the ballooning equation on each field line, labeled by $q$ and $\alpha$, and for each value of $\theta_{k}$, giving $\lambda=\lambda\left(q, \alpha, \theta_{k}\right)$. Thus we use the ballooning equation to give us a local dispersion relation

$$
\omega^{2}=\lambda\left(q, \alpha, \theta_{k}\right)
$$

relating the frequency $\omega$ (a global constant) and the wavevector, through the relation $\theta_{k}=$ $k_{q} / k_{\alpha}$, on each field line. Here we have changed variables so that $q$ replaces $s$ as radial variable, assuming nonzero global magnetic shear everywhere.

The ray equations ${ }^{8}$ can be reduced to

$$
\begin{aligned}
\dot{\alpha} & =-\theta_{k} \frac{\partial \lambda}{\partial \theta_{k}}, \\
\dot{q} & =\frac{\partial \lambda}{\partial \theta_{k}}, \\
\dot{\theta_{k}} & =\theta_{k} \frac{\partial \lambda}{\partial \alpha}-\frac{\partial \lambda}{\partial q} .
\end{aligned}
$$


In this paper we are concerned with modes trapped in a $\lambda$-well in each $q-\theta_{k}$ section, whose rays thus perform bounded oscillations in $q$ and $\theta_{k}$. Due to the $L$-fold toroidal periodicity of stellarator equilibria ( $L$ being the number of field periods), there will be periodic minima of $\lambda$, spaced $2 \pi / L$ apart in $\alpha$, with periodic saddle points occurring between these minima. Let the value of $\lambda$ at the minima be $\lambda_{0}$ (negative for instability) and the value at the saddles be $\lambda_{x}$. For $\lambda_{0}<\omega^{2}<\lambda_{X}$ the level surfaces of $\lambda$ defined by Eq. (18) are spheroidal, highly elongated in the $\alpha$ direction. The rays propagate between the unstable and stable fixed points of the dynamical system defined by Eqs. (19)-(21). Geometrically, the fixed points correspond to the points on the level surfaces where the normal is parallel to the $\left(q, \alpha, \theta_{k}\right)$ space vector $\left(\theta_{k}, 1,0\right)$. In Ref. 8 the range $\lambda_{0}<\omega^{2}<\lambda_{X}$ over which the rays were attracted to fixed points was interpreted as an unstable continuum band.

For $\omega^{2}>\lambda_{X}$ the level surfaces are bumpy cylinders and the rays propagate secularly downwards in $\alpha$, as illustrated in Fig. 1. Figure 2 shows the projection of a ray trajectory onto the $\left(q, \theta_{k}\right)$ plane. It is seen that the ray traces out a cyclic motion. Introducing an angle $\phi=\operatorname{Arg}\left[\left(q-q_{0}\right)+i\left(\theta_{k}-\theta_{k 0}\right)\right]$ [where $\left(q_{0}, \theta_{k 0}\right)$ is an arbitrary point interior to the envelope of the ray projection in the $\left(q, \theta_{k}\right)$ plane, see Fig. 2], which increases by $2 \pi$ each counter-clockwise $q-\theta_{k}$ circuit, we define a rotation number $\nu \equiv \nu\left(\omega^{2}\right)$ for the rays as the mean rate of increase, with respect to $\phi$, of $\alpha$

$$
\nu=\lim _{\phi \rightarrow \infty} \frac{\alpha-\alpha_{0}}{\phi-\phi_{0}} .
$$

Note that, since both $\alpha$ and $\phi$ have a secular component decreasing along a ray, $\nu$ is positive. In the axisymmetric case ${ }^{7}$

$$
\nu=\frac{1}{2 \pi} \oint \theta_{k} d q
$$

where the integral is taken around the contour defined by Eq. (18). In this case $\nu$ is a monotonic function of $\omega^{2}$, but in the general three-dimensional case there is a mode-locking phenomenon which leads to a devil's staircase structure. ${ }^{8}$ However, we find the widths of the steps (which occur at each rational value of $\nu$ ) to be extremely narrow so that $\nu\left(\omega^{2}\right)$ is still a smooth function for practical purposes, even in the three-dimensional case. 
The phase $S / \epsilon$ is constant on a ray except when its projection in the $q-\alpha$ plane passes through a caustic, ${ }^{8}$ where it jumps by $\pi / 2$; a caustic being a boundary of the projection in the $q-\alpha$ plane of the set of all rays corresponding to a given $\omega^{2}-$ i.e. a line of reflection points. In the $q-\alpha$ plane the problem is analogous to propagation of a mode in a planar waveguide with periodically modulated boundary. With the additional toroidal periodicity condition, ${ }^{8}$

$$
\epsilon^{-1}[S(\alpha+2 \pi, q)-S(\alpha, q)]=-2 \pi n,
$$

we find the semiclassical quantization condition

$$
\nu\left(\omega^{2}\right)=\frac{2 N+1}{2 n},
$$

where $N=0,1,2, \ldots$ is the radial mode number and $n=1,2, \ldots$ is the toroidal mode number.

Typically $N$ is taken to be zero (the lowest radial eigenmode) to maximize the growth rate, though for strict validity of the asymptotics and, to give a trapped mode macroscopic extent, it should be taken $O(n)=O\left(\epsilon^{-1}\right)$. Another reason for considering $N \neq 0$ is the interesting degeneracy implied by the form of Eq. (25) - there is an infinite set of $(n, N)$ pairs giving the same growth rate.

\section{NUMERICAL WKB STUDY}

Given a differentiable representation of $\lambda\left(q, \alpha, \theta_{k}\right)$, the integration of the ray equations, Eqs. (19)-(21), is straightforward (the LSODE package is used here ${ }^{19}$ ). We evaluate $\lambda$ on a $\left(q, \alpha, \theta_{k}\right)$-lattice and use a piecewise quadratic, globally $C^{1}$ interpolating function such that the derivative values used in Eqs. (19)-(21) are analytically consistent with the interpolating function $\lambda_{I}\left(q, \alpha, \theta_{k}\right)$. These properties are essential if the ray integrator is to maintain rays on an isosurface of Eq. (18) (a defining property of the ray equations). We have found it possible to achieve variations in $\lambda$ along a ray of less than $0.001 \%$. The $\left(q, \alpha, \theta_{k}\right)$-lattice 
covering one field period and magnetic surfaces on which the eigenvalue is positive was $63 \times 24 \times 60$.

The evaluation of $\lambda$ on the lattice points could be done in parallel by allocating these points to processors and solving each problem independently, entirely on one processor. However for a SIMD computer such as the CM5 used here, it is more efficient to distribute each eigenproblem across all processors and sweep through the lattice points sequentially. The main reason for this is that, as a linear system, the ballooning equations are amenable to a generalized parallel prefix solution method, an $O\left(\log \left(N_{p}\right)\right)$ solution where $N_{p}$ is the number of points along the field line. ${ }^{20}$

\section{EQUILIBRIUM AND GLOBAL STABILITY}

The preconditioned VMEC equilibrium code ${ }^{21}$ is employed to numerically compute a 3-D equilibrium state for an $L=10$ field period torsatron model. The code imposes perfectly nested magnetic flux surfaces. The pressure profile, $p(s)=p(0)\left(1-s^{2}\right)^{2}$, is chosen to be flat near the magnetic axis so as to have a large pressure gradient outside the magnetic well region in order to create a ballooning unstable situation, taking $\beta_{0}$ (the value of $\beta$ on axis) to be $4 \%$. The toroidal current profile was prescribed to vanish within each flux surface. The spectrum of Fourier modes used in the calculation encompassed 72 active mode pairs in the optimal but nonstraight field line coordinates of the VMEC code. This configuration has been established as a testbed for a comparison of a number of 2D and 3D MHD stability codes and a much more detailed description of the equilibrium computation and the low-n stability properties has been reported by Nakamura et al. ${ }^{22}$

The configuration is unstable to both local (infinite $n$ ) and to global (low $n$ ) ideal MHD modes at $\beta_{0}=4 \%$ with the current and pressure profiles prescribed. The global stability calculations were carried out with the TERPSICHORE code ${ }^{11}$ with a conducting wall placed at the plasma-vacuum interface. A very efficient Fourier technique was employed to reconstruct the equilibrium in the magnetic coordinates, ${ }^{23}$ with the spectrum of Fourier 
mode pairs increased to 194 . The number of poloidal grid points was 120 and the number of toroidal grid points per field period was 24. To examine stability properties, 94 Fourier mode pairs were selected to describe the instability structure, of which 30 corresponded to the toroidal mode number $n_{0}$ specifically under investigation and the remaining 64 mode pairs were associated with the most relevant toroidal sidebands of the components that dominate.

The mode selection criteria are simplified because the conditions of stellarator symmetry and the finite number of field periods imply the existence of families of Fourier modes that decouple from each other, in analogy with the condition in axisymmetry that decouples each toroidal Fourier mode number. A Fourier mode family labeled by the integer $1 \leq K \leq L / 2$ involves all integer poloidal Fourier mode numbers, $m$, (which may be restricted to $m \geq 0$ by using the reality condition) but its toroidal Fourier mode numbers $n$ are drawn from the set $\left\{L N_{\mathrm{e}} \pm K \mid N_{\mathrm{\varepsilon}}=0, \pm 1, \pm 2, \ldots\right\}$, where $L$ is the number of equilibrium field periods (10 in our case). ${ }^{24}$ This terminology was subsequently adopted for advanced stellarator configurations where the family index $K$ has been extended to include the equilibrium state also. ${ }^{14}$ An essential feature of the global stability computations was the identification of the principal resonant components of the instability structure for a desired value of the toroidal mode number $n_{0}$ and the inclusion of the nonresonant toroidal sidebands that couple them to beat with the main symmetry-breaking Fourier components that describe the equilibrium state. This was a necessary step to guarantee not only a quantitative but also a qualitative agreement of the eigenvalues computed with TERPSICHORE and those predicted with WKB-ballooning theory. This constituted a nontrivial exercise, especially for the larger$N$ radial eigenmodes because, for example, instability structures dominated by the $n=8$ component belong to the same family as those dominated by the $n=2$ and the $n=12$ components for a 10 -field-period configuration, namely the $K=2$ family.

Using TERPSICHORE, the three most unstable eigenvalues were computed for each $n$ in the range $2 \leq n \leq 14$, except for $n=L / 2=5$ and $n=L=10$. These three eigenvalues correspond to the radial eigenmodes $N=0$ (most unstable), $N=1$, and $N=2$. The 
eigenvalues were converged to infinitesimal mesh based on calculations with $108,128,152$, and 192 radial intervals in the plasma domain.

A comparison of the results of the WKB-ballooning ray tracing method with the TERP. SICHORE results described in this section is presented in Fig. 3. The numerical ray tracing code described in Sec. III was used to calculate $\nu$ from Eq. (22) [and also from Eq. (23) with very similar results] for a set of $\omega^{2}$ values. Then Eq. (25) was used to predict $n$, taking $N=0,1,2$. This gave the three curves shown in Fig. 3 . The fixed-point continuum band described in Sec. II lies between $-\lambda=0.004187$ and $-\lambda=0.004176$, which is too narrow to resolve on the scale of the graph and lies well above the eigenvalues found.

It is seen that the presence of these three unstable branches was verified by TERPSICHORE with remarkable accuracy, given that the WKB formalism is strictly valid only for large $n$ and $N$. Also, uncertainties in the converged TERPSICHORE eigenvalues can be up to about \pm 0.0001 .

\section{CONCLUSIONS}

By successfully comparing two very different methods for calculating stellarator stability we have provided validation for both. The global eigenvalue approach avoids the small wavelength approximation needed for WKB-ballooning, but requires large amounts of computer memory and careful convergence studies. The WKB-ballooning approach is easier to generalize by adding more physics, and is the natural method when the modes of interest are indeed of short wavelength, though we have also shown that it works well for quite small mode numbers. It is planned in future to study more strongly nonaxisymmetric cases than the one in the present paper.

\section{ACKNOWLEDGMENTS}

We acknowledge with gratitude Drs H.J. Gardner and M. Persson for useful discussons. The ballooning calculations were carried out on the Thinking Machines Corporation CM-5 
of the Parallel Computing Research Facility of the Centre for Information Science Research, Australian National University. The work of one of us (WAC) was partially sponsored by the Fonds National Suisse de la Recherche Scientifique and by Euratom. We thank Dr. S.P. Hirshman for use of the VMEC equilibrium code. 


\section{REFERENCES}

${ }^{1}$ J.W. Connor, R.J. Hastie, and J.B. Taylor, Proc. R. Soc. London A 365, 1 (1979).

2 J.W.Connor, J.B. Taylor, and H.R. Wilson, Phys. Rev. Lett. 70, 1803 (1993).

${ }^{3}$ F. Romanelli and F. Zonca, Phys. Fluids B 5, 4081 (1993).

J.Y. Kim and M. Wakatani, Phys. Rev. Lett. 73, 2200 (1994).

${ }^{5}$ R.L. Dewar, Y.Z. Zhang, and S.M. Mahajan, Phys. Rev. Lett. 74, 4563 (1995).

${ }^{6}$ Y.Z. Zhang, S.M. Mahajan, and X.D. Zhang, Phys. Fluids B 4, 2729 (1992).

${ }^{7}$ R.L. Dewar, J. Manickam, R.C. Grimm, and M.S. Chance, Nucl. Fusion 21, 493 (1981); Corrigendum Nucl. Fusion 22, 307 (1982).

${ }^{8}$ R.L. Dewar and A.H. Glasser, Phys. Fluids 26, 3038 (1983).

${ }^{9}$ R.L. Dewar, Ballooning mode Schrödinger equation in "Theory of Fusion Plasmas," A. Bondeson, E. Sindoni, and F. Troyon eds. (Soc. Ital. Fisica - Editrice Compositori, Bologna, 1988) pp. 107-122.

${ }^{10}$ R.L. Dewar and Y.Z. Zhang, Bull. Am. Phys. Soc. 37, 1436 (1992).

${ }^{11}$ D.V. Anderson, W.A. Cooper, R. Gruber, S. Merazzi, and U. Schwenn, Int. J. Supercomp. Appl. 4, 34 (1990).

${ }^{12}$ I.B. Bernstein, E.A. Frieman, M.D. Kruskal, and R.M. Kulsrud, Proc. R. Soc. London A 244, 17 (1958).

${ }^{13} \mathrm{~J}$. Nührenberg and R. Zille, Equilibrium and stability of low-shear stellarators in "Theory of Fusion Plasmas," A. Bondeson, E. Sindoni, and F. Troyon eds. (Soc. Ital. Fisica Editrice Compositori, Bologna, 1988) pp. 3-23.

${ }^{14}$ C. Schwab, Phys. Fluids B5, 3195 (1993).

15 J. Manickam, R.L. Dewar, and R.C. Grimm, Comput. Phys. Commun. 24, 355 (1981). 
${ }^{16}$ G.Y. Fu, W.A. Cooper, R. Gruber, S.Merazzi, and U. Schwenn, Phys. Fluids B4, 1401 (1992).

${ }^{17}$ A.H. Boozer, Phys. Fluids 23, 904 (1980).

${ }^{18}$ W.A. Cooper, Plasma Phys. Contr. Fusion 34, 1011 (1992).

${ }^{19}$ A.C. Hindmarsh, ODEPACK, a systematized collection of ode solvers, in Scientific Computing, R.S. Stepleman et al. (eds.) (North-Holland, Amsterdam, 1983) pp. 55-64.

${ }^{20}$ G.E. Blelloch, Vector Models for Data-Parallel Computing (MIT Press, Cambridge, MA, 1990).

${ }^{21}$ S.P. Hirshman and O. Betancourt, J. Comput. Phys. 96, 99 (1991).

${ }^{22}$ Y. Nakamura, T. Matsumoto, M. Wakatani, S.A. Galkin. V.V. Drozdov, A.A. Martynov, Yu.Yu. Poshekhnonov, K. Ichiguchi, L. García, B.A. Carreras, C. Nührenberg (née Schwab), W.A. Cooper, and J.L. Johnson, Comparison of the calculations of the stability properties of a specific stellarator equilibrium with different MHD stability codes, submitted to J. Comput. Phys.; Princeton Plasma Physics Laboratory Report PPPL-3092 (1995).

${ }^{23}$ D.V. Anderson, A. Cooper, U. Schwenn, and R. Gruber, Linear MHD stability analysis of toroidal SD equilibria with TERPSICHORE in "Joint Varenna-Lausanne International Workshop on Theory of Fusion Plasmas," J. Vaclavik, F. Troyon, and E. Sindoni eds. (Soc. Ital. Fisica - Editrice Compositori, Bologna 1989) pp. 93-102.

${ }^{24}$ W.A. Cooper, G.Y. Fu, R. Gruber, S. Merazzi, U.Schwenn, and D. V. Anderson, Global external ideal magnetohydrodynamic instabilities in three-dimensional plasmas in "Joint Varenna-Lausanne International Workshop on Theory of Fusion Plasmas," E. Sindoni, F. Troyon, and J. Vaclavik eds. (Soc. Ital. Fisica - Editrice Compositori, Bologna 1990) pp. 655-665. 


\section{FIGURES}

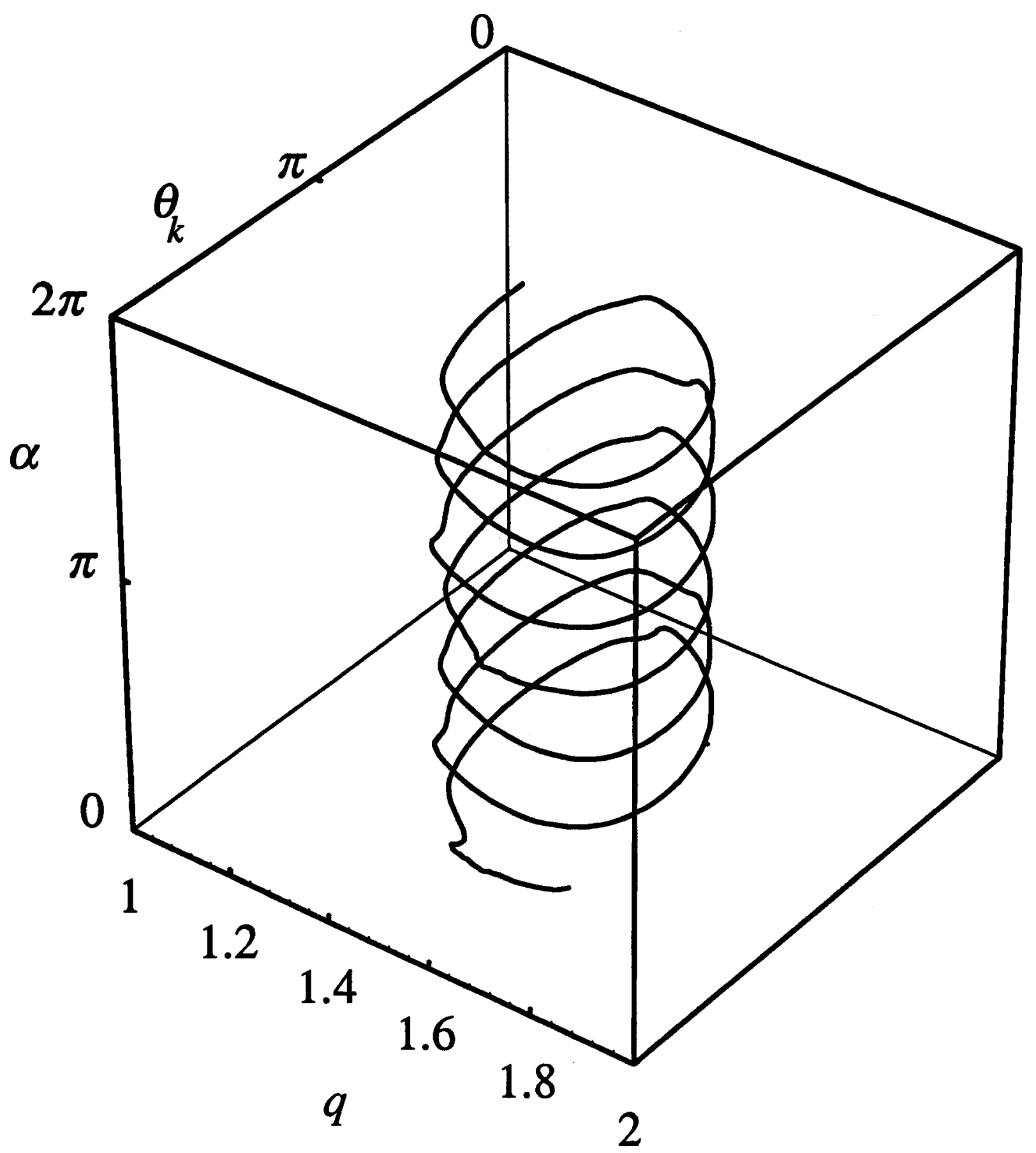

FIG. 1. Typical ray trajectory in $\left(q, \alpha, \theta_{k}\right)$ space. 


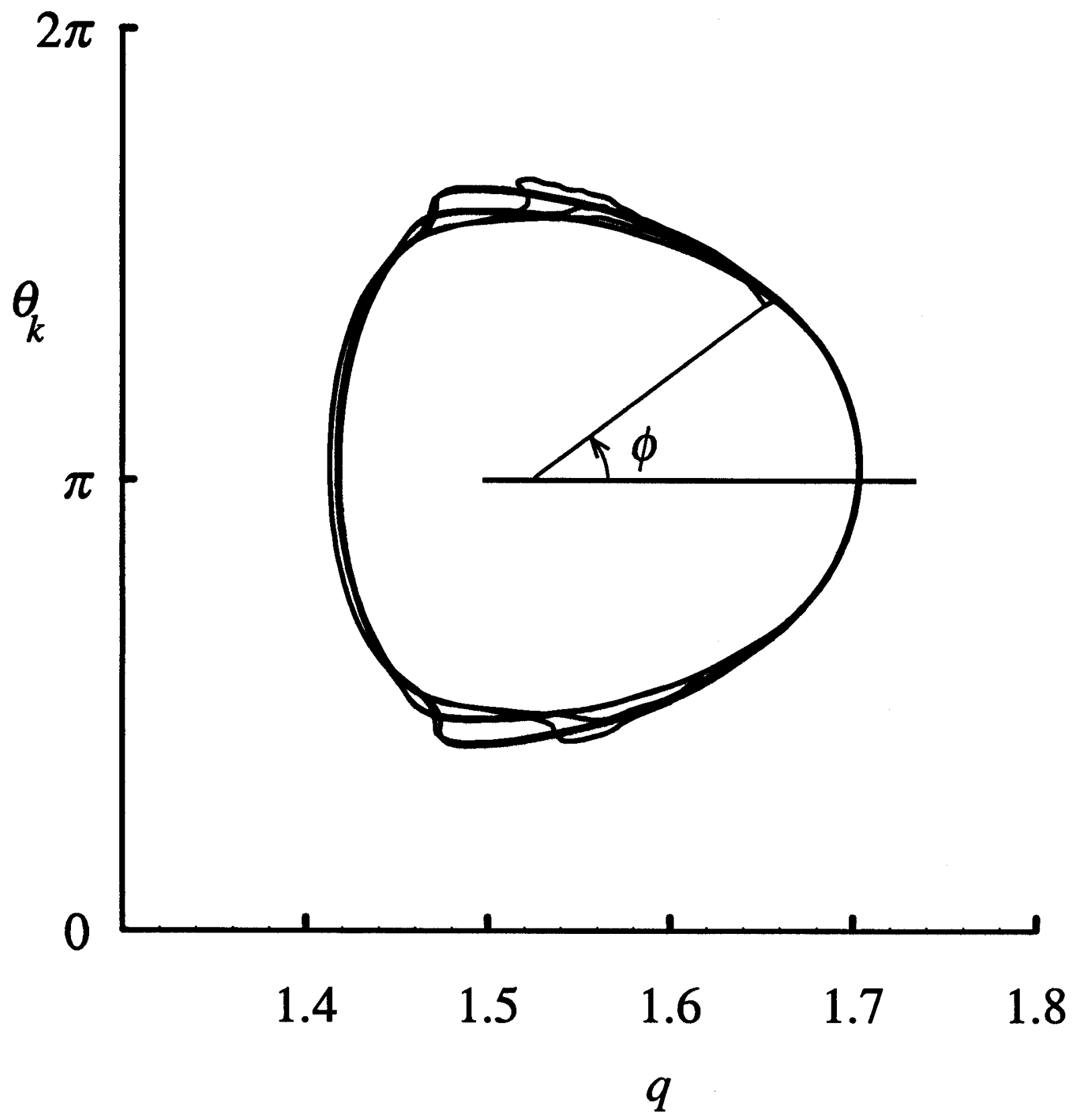

FIG. 2. Projection of the ray trajectory of Fig. 1 onto the $\left(q, \theta_{k}\right)$ plane. Also shown is the angle $\phi$ used to define the ray rotation number $\nu$. 


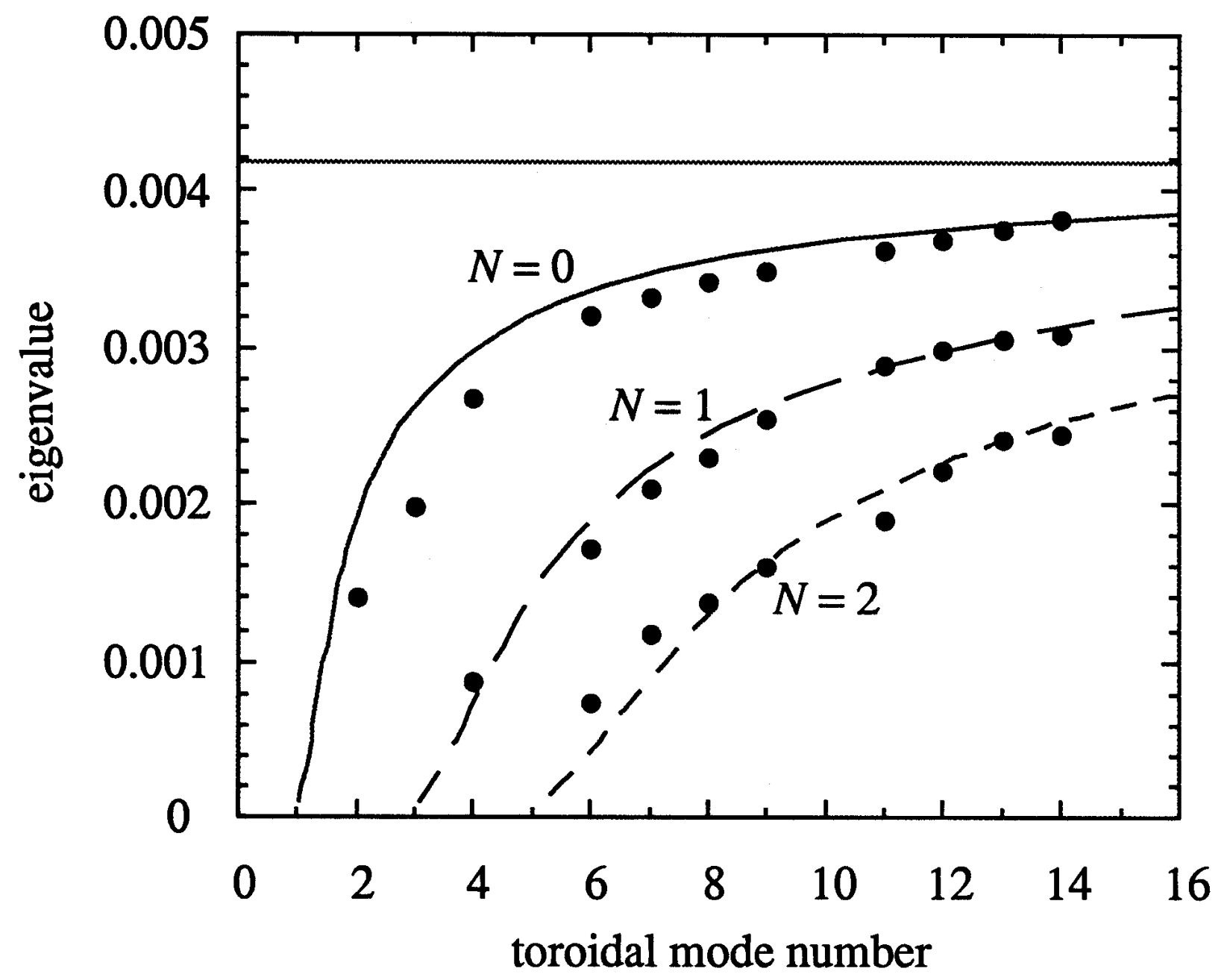

FIG. 3. Comparison of WKB-ballooning growth rate eigenvalues, $-\omega^{2}$, (curves) with TERPSICHORE results (dots) plotted vs. dominant toroidal mode number $n$ for the first three radial mode numbers $N=0,1,2$. The gray horizontal line represents the $n=\infty$ continuum band defined by fixed points of the ray equations (see text). 